\title{
Experimental Test of Nuclear Magnetization Distribution and Nuclear Structure Models
}

\author{
P. Beiersdorfer, J. R. Crespo Lopez-Urrutia, S. B. Utter
}

February 26, 1999

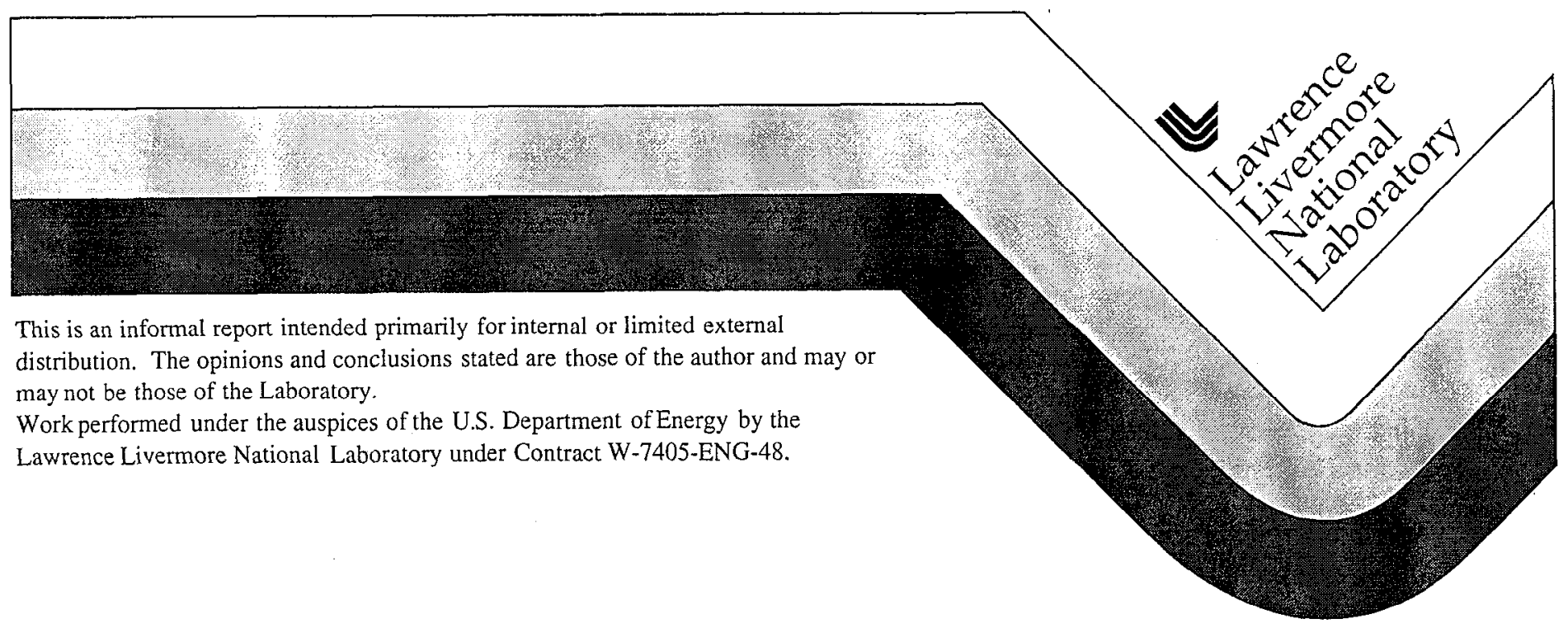




\section{DISCLAIMER}

This document was prepared as an account of work sponsored by an agency of the United States Government. Neither the United States Government nor the University of California nor any of their employees, makes any warranty, express or implied, or assumes any legal liability or responsibility for the accuracy, completeness, or usefulness of any information, apparatus, product, or process disclosed, or represents that its use would not infringe privately owned rights. Reference herein to any specific commercial product, process, or service by trade name, trademark, manufacturer, or otherwise, does not necessarily constitute or imply its endorsement, recommendation, or favoring by the United States Government or the University of California. The views and opinions of authors expressed herein do not necessarily state or reflect those of the United States Government or the University of California, and shall not be used for advertising or product endorsement purposes.

This report has been reproduced directly from the best available copy.

Available to DOE and DOE contractors from the Office of Scientific and Technical Information

P.O. Box 62, Oak Ridge, TN 37831

Prices available from (615) 576-8401, FTS 626-8401

Available to the public from the

National Technical Information Service

U.S. Department of Commerce

5285 Port Royal Rd.,

Springfield, VA 22161 


\title{
Experimental test of nuclear magnetization distribution and nuclear structure models
}

\author{
98-LW-057 Final Report
}

\author{
P. Beiersdorfer, J. R. Crespo López-Urrutia, and S. B. Utter
}

A correct description of the internal composition of an atomic nucleus is fundamental to our understanding of the building blocks that make up our everyday universe. While we know such basic characteristics as the number of protons and neutrons in the nucleus of each element and isotope, we have a more limited understanding of the actual arrangement of these particles and their interaction. Much of our understanding of nuclear structure has come from scattering experiments. For example, bombarding a nucleus with electrons and counting the number electrons deflected by varying degrees gives detailed information on the arrangement of protons and the associated distribution of electric charge in the nucleus. Similarly, bombarding the nucleus with magnetic monopoles and measuring the amount of scatter would give us information on the distribution of currents and magnetic fields in the nucleus. The problem, however, is that no magnetic monopoles are available to carry out such experiments. In fact, it is not clear that they even exist. As a result, our understanding of collective effects in the nucleus, such as nuclear currents and the distribution of magnetic fields within the nucleus, are very limited. Models exist that ascribe the nuclear magnetic fields to the presence of a single nucleon whose spin is not neutralized by pairing it up with that of another nucleon; other models assume that the generation of the magnetic field is shared among some or all nucleons throughout the nucleus. All models predict the same magnetic field external to the nucleus since this is an anchor provided by experiments. The models differ, however, in their predictions of the magnetic field arrangement within the nucleus for which no data exist. The only way to distinguish which model gives the correct description of the nucleus would be to use a probe inserted into the nucleus. The goal of our project was to develop exactly such a probe and to use it to measure fundamental nuclear quantities that have eluded experimental scrutiny. The need for accurately knowing such quantities extends far beyond nuclear physics and has ramifications in parity violation experiments on atomic traps and the testing of the standard model in elementary particle physics.

Unlike scattering experiments that employ streams of free particles, our technique to probe the internal magnetic field distribution of the nucleus rests on using a single bound electron. Quantum mechanics shows that an electron in the innermost orbital surrounding the nucleus constantly dives into the nucleus and thus samples the fields that exist inside. This sampling of the nucleus usually results in only minute shifts in the electron's average orbital, which would be difficult to detect. By studying two particular energy states of the electron, we can, however, dramatically enhance the effects of the distribution of the magnetic fields in the nucleus. In fact about $2 \%$ of the energy difference between the two states, dubbed the hyperfine splitting, is determined by the effects related to the distribution of magnetic fields in the nucleus. A precise measurement of this energy difference (better than $0.01 \%$ ) would then allow us to place stringent bounds on the models predicting currents and magnetic fields in the nucleus.

We have implemented our method by constructing a very high-resolution spectrometer sensitive to light near $3800 \AA$, which is the wavelength of light corresponding to the hyperfine 
splitting of the orbital of a single electron bound to a thallium nucleus, $\mathrm{Tl}^{80+}$. The spectrometer is unique in its design and consists of two independent arms separated by $90^{\circ}$, each with an ultra sensitive CCD camera, a large-diameter transmission grating, and various focusing elements (see schematic drawing, Fig. 1). The gratings were manufactured using Laser Program facilities and leveraging unique Livermore expertise in manufacturing of high-throughput optics, described by Nguyen et al. in [1]. The two identically manufactured gratings are 6 inch diameter and have line densities of 2850 lines $/ \mathrm{mm}$. The groove depth and shape are optimized for $3800 \AA$ light. One leg consists of a matched pair of $130 \mathrm{~mm}$ diameter $f / 4.6$ achromatic lenses, whereas the other leg utilizes $100 \mathrm{~mm}$ diameter $f / 4.0$ achromats. Otherwise, the two legs are identical. The source is positioned at the focus of the first, collimating, optic so as to produce parallel 'pseudowhite' light. The emitted light is dispersed by the high-efficiency transmission grating, and a portion of this is collected by the focusing optic used to focus the wavelengths of interest at the $\mathrm{CCD}$ detector. The detector is mounted on a linear translation stage making adjustments along the dispersion direction possible. All of the spectrometer components are mounted to an optics table made light tight with black anodized aluminum sheet walls and lid. On the Super EBIT system, high-efficiency $f / 2$ optics are inserted inside the vacuum vessel producing an image of the source outside of the chamber. This allows for easier access by the large spectrometer housing, and is required for access with the $f / 4.0$ collimating optic of the second leg. The two legs of the system compliment each other by effectively doubling the signal count rate while providing two independent measurements.

The SuperEBIT facility is the only device capable of producing $\mathrm{Tl}^{80+}$ ions in sufficient quantities to carry out the planned measurements. However, unrelated to our project, the machine was completely incapacitated in May 1998 by a failure of the vacuum and cooling systems. Repairs are currently underway, but a completion date is still unknown. The successful completion of our measurements must now await the resumption of operations on this facility.

In spite of this, the spectrometer was successfully tested at the Livermore EBIT facility using the lower energy device, EBIT-II, and found to produce results to an accuracy of about 1 part in 65,000 with typical line widths on the order of 5.5 channels of the CCD detector, or equivalently, $0.55 \AA$. Measurements of neutral and near-neutral charge state transitions of both neon and krypton were made throughout the range of $3500 \AA$ to $4000 \AA$. These lines are well known and sufficiently accurate for wavelength calibration [2]. To demonstrate the capabilities of this spectrometer we have made the first measurement of the $3 \mathrm{~d}^{4}{ }^{5} \mathrm{D}_{2}-{ }^{5} \mathrm{D}_{3}$ transition in Ti-like $\mathrm{W}\left(\mathrm{W}^{52+}\right)$ near $3650 \AA$. This transition is of interest as a high-temperature plasma diagnostic due to a very unusual characteristic: the wavelength of this transition is expected to remain in the visible to the near-UV over a broad range of mid- $Z$ ions [3]. Once these transition energies have been measured a single spectrometer will be able to be used to measure the plasma temperature in a high temperature fusion device over a large range of temperatures by scanning for this transition throughout the range of ions. Accurate measurements may also be useful in assisting the improvement of calculations to predict further transition energies along the isoelectronic sequence. Figure 2 shows a series of spectra taken at one spectrometer setting in the region near 3600 to $3700 \AA$. Figures $2 \mathrm{a}$ and $2 \mathrm{~b}$ display the calibration spectra of neutral and near-neutral krypton and neon, respectively. The spectra provide a high density of calibration lines for excellent anchoring of the wavelength scale. Figure $2 c$ shows the single line of $W$ which was found to go away when EBIT was dropped below the ionization threshold to produce $\mathrm{W}^{52+}$. A search along the dispersion direction revealed no other lines produced by tungsten in the range of 3500 to $4200 \AA$. 
Despite the breakdown of SuperEBIT, 98-LW-057 was successful in creating new capabilities unique to LLNL. High-precision measurements in the near-UV domain made possible with the development of this unique spectrometer system, coupled with the diverse capabilities of an electron beam ion trap, provide a pathway for the undertaking of scientifically and programmatically interesting measurements that test our fundamental knowledge of the physical world, such as the nuclear magnetization measurements we set out to do, as well as provide necessary data for the understanding of high-temperature plasmas.

This work was performed by LLNL under the auspices of the United States Department of Energy under contract W-7405-ENG-48.

References:

[1] "High-efficiency fused-silica transmission gratings", H. T. Nguyen, B. W. Shore, S. J. Bryan, J. A. Britten, R. D. Boyd, and M. D. Perry, Opt. Lett. 22, 142 (1997).

[2] "Wavelengths and transition probabilities for atoms and atomic ions", J. Reader, C. Corliss, W. Wiese, and G. Martin, U. S. Dept. of Commerce, National Bureau of Standards, NSRDS-NBS 68 (1980).

[3] "Magnetic dipole line from U-LXXI ground-term levels predicted at $3200 \AA$ ", U. Feldman, P. Indelicato, and J. Sugar, J. Opt. Soc. Am. B 8, 3 (1991). 
Figure Captions:

Figure 1: Schematic diagram of one leg of the spectrometer system. Radiation from trapped ions in EBIT is emitted through a quartz window on a side port. A collimating lens with a large aperture renders this light parallel. The high-efficiency quartz transmission grating disperses the light, a portion of which is collected by the focusing lens. A range of wavelengths is then detected and stored by the CCD chip.

Figure 2: A sample of the data collected by the CCD for the measurement of the $3 \mathrm{~d}^{4}{ }^{5} \mathrm{D}_{2}-{ }^{5} \mathrm{D}_{3}$ transition in Ti-like W $\left(\mathrm{W}^{52+}\right)$. (a) and (b) Spectra obtained due to the injection of krypton and neon, respectively. These neutral and near-neutral lines are used as calibration for determining the transition energy of the line of interest. (c) Spectrum resulting from tungsten injection at a beam energy of $5.1 \mathrm{keV}$, the optimal energy for the production of Ti-like $\mathrm{W}$. This line was found to disappear at energies lower than those necessary to produce Ti-like $\mathrm{W}$, thereby confirming its association with that charge state. 
TRANSMISSION GRATING

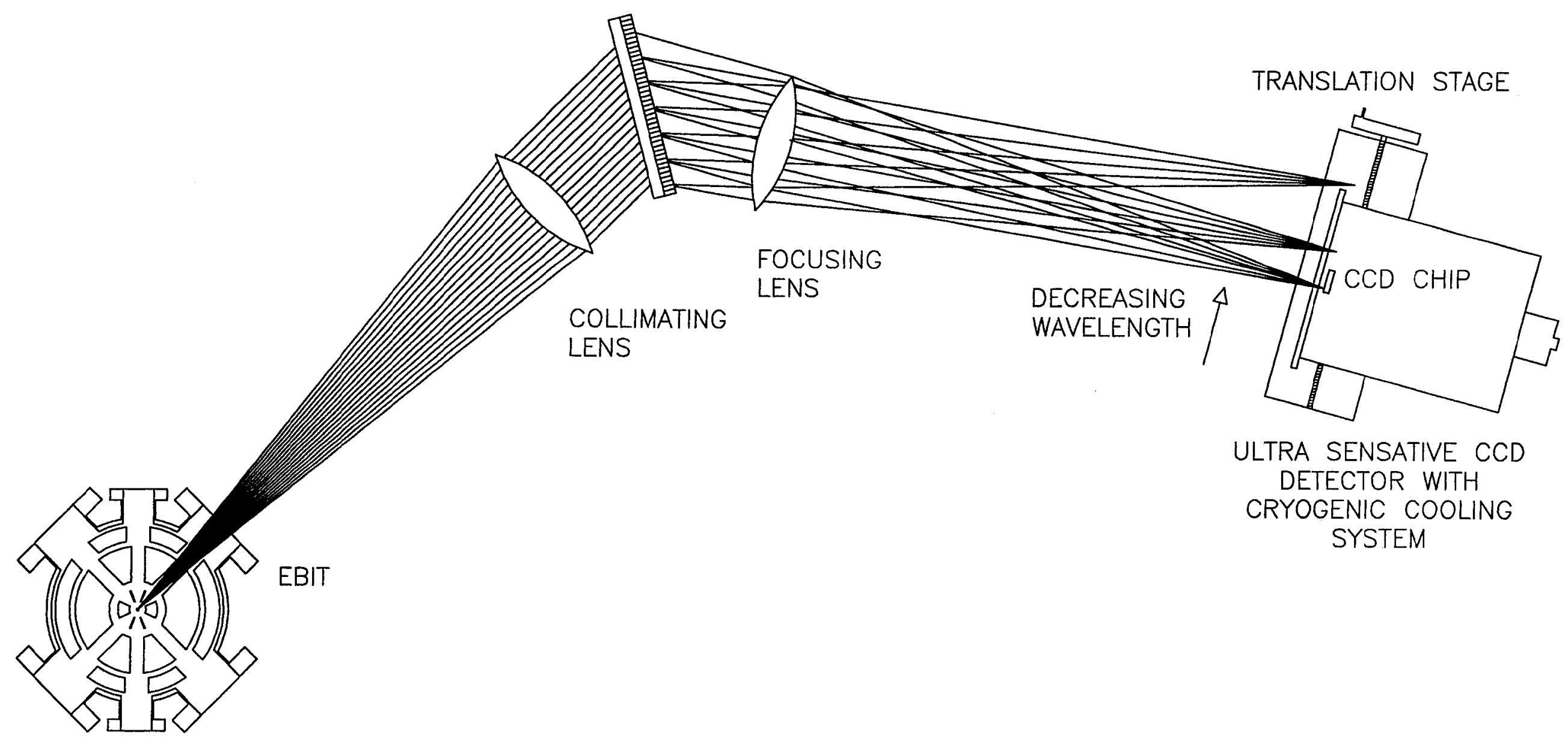




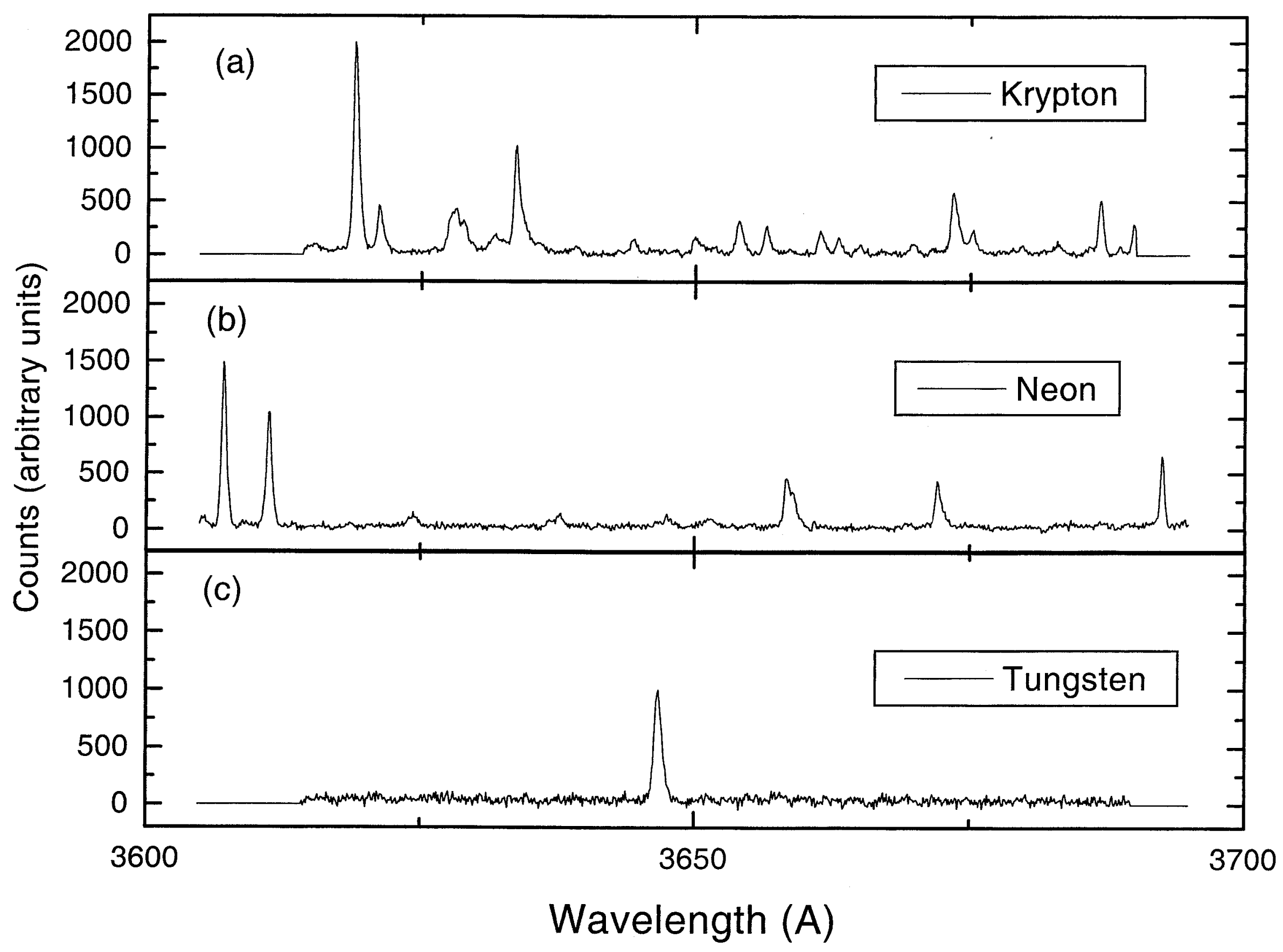

Figure 2. 